\title{
Low dietary calcium intake does not modify fracture risk but increases fall frequency: the results of GO Study
}

\author{
Wojciech Pluskiewicz', Piotr Adamczyk ${ }^{2}$, Bogna Drozdzowska ${ }^{3}$ \\ ${ }^{1}$ Department and Clinic of Internal Diseases, Diabetology, and Nephrology, Metabolic Bone Diseases Unit, Faculty of Medical \\ Sciences in Zabrze, Medical University of Silesia in Katowice, Poland \\ ${ }^{2}$ Department of Paediatrics, Faculty of Medical Sciences in Katowice, Medical University of Silesia in Katowice, Poland \\ ${ }^{3}$ Department of Pathomorphology, Faculty of Medical Sciences in Zabrze, Medical University of Silesia in Katowice, Poland
}

\begin{abstract}
Introduction: The aim of the study was to verify the thesis that dietary calcium intake influences the risk of osteoporotic fractures established by online available calculators.

Material and methods: The study was performed in 521 postmenopausal women aged over 55 years, recruited in one osteoporotic outpatient clinic. Mean age was $67.7 \pm 8.6$ years. Fracture risk was established using FRAX (major and hip fractures, 10 years), Garvan calculator (any and hip fractures, 5 and 10 years), and the Polish algorithm available at www.fracture-risk.pl (any fractures, 5 years). Bone densitometry at the femoral neck was performed using a DPX device (Lunar, GE, USA) to calculate fracture risk by each of those calculators. Calcium intake was established based on a dietary questionnaire.

Results: Mean values of fracture risk for all three calculators and T-score value for DXA measurement at the femoral neck did not correlate with calcium intake. A tendency to insignificantly lower calcium intake was observed in the subgroup with high hip fracture risk by FRAX ( $\geq 3 \%$ ) vs. low hip FRAX (<3\%): $744 \pm 328 \mathrm{mg}$ /day vs. $765 \pm 299 \mathrm{mg} /$ day. The same analysis for FRAX major fracture risk revealed a similar tendency: $700 \pm 299 \mathrm{mg}$ /day and $760 \pm 311 \mathrm{mg} /$ day in high $(\geq 20 \%)$ and low $(<20 \%)$ fracture-risk groups, respectively. Calcium intake did not influence the results obtained in the other two calculators at all. Calcium intake did not differ between subjects with prior falls and those without falls. However, if the number of falls was taken into account, the women who reported three and more falls had significantly lower calcium intake $(621 \pm 275 \mathrm{mg} /$ day $)$ than subjects with no falls $(767 \pm 304 \mathrm{mg} / \mathrm{day}$; $\mathrm{p}<0.05)$ or those with one fall $(766 \pm 317 \mathrm{mg} /$ day; $\mathrm{p}<0.05)$.

Conclusions: Calcium intake does not correlate with fracture risk established by calculators available on-line, but low calcium intake may increase the risk of falls. (Endokrynol Pol 2021; 72 (3): 198-201)
\end{abstract}

Key words: calcium intake; falls; fracture risk

\section{Introduction}

The pathogenesis of involutional osteoporosis involves several different factors that cause bone loss. Age-related changes in the endocrine system, secondary senile hyperparathyroidism, physical activity, diet, some medications, or increasing morbidity constitute this factor spectrum. In regard to the role of diet, calcium intake is the most important issue. In a review on the role of calcium for bone health it was clearly stated that adequate calcium intake throughout one's lifetime is important for bone health, and an adequate calcium supply reduces bone loss by $0.5-1.2 \%$ and the risk of all types of fractures by at least $10 \%$ in the elderly [1]. Other authors demonstrated that increased calcium intake produces small non-progressive increases in bone mineral density (BMD), which are unlikely to lead to a clinically significant reduction in the risk of fracture [2]. In a prospective observation of a large cohort of
41,514 subjects for a period of 12 years, higher calcium intake was associated with reduced risk of fractures [3]. Similar results in regard to spine fractures were presented by Italian authors [4]. Also, another longitudinal observation confirmed that low calcium intake (lowest quintile) increases fracture risk [5]. A review and meta-analysis of 33 randomized clinical trials summarized that a relationship between calcium intake and fracture risk does not exist [6]. Another expert consensus stated that supplementation with calcium alone for fracture reduction is not supported by literature, and calcium plus vitamin D leads to modest reduction of fracture risk [7]. Some authors verified the thesis that calcium may enhance BMD; contradictory results were also obtained - a recent Australian study revealed a positive relationship between calcium intake and femoral neck BMD [8], while other authors did not observe such a relationship [9]. In our study, performed in a population-based cohort of postmenopausal women over 55 years of age, 
calcium intake was lower in those with fractures and in those with low femoral neck BMD [10]. Very convincing evidence of a positive role of adequate calcium supply on human health was presented in the aforementioned study [3], which documented not only the reduction in fracture risk but also a decrease in the risk of non-fatal cardiovascular events, stroke, and all-cause mortality.

All presented data show discrepancies in the results of different studies and provide the final conclusion that the role of calcium intake on bone health is not clearly established and further studies are necessary. Moreover, the relation between calcium and fracture incidence or BMD measurements may be also modified indirectly by potential the influence of calcium on different aspects of human health. The important role of calcium in many biological mechanisms may contribute to combined effects also on fracture risk. For example, it has been shown that calcium regulation disorders can lead to vertigo or syncope through cochlear [11] or cardiac [12] mechanisms. This in turn can clearly result in falls, injuries, and low-energy fractures.

In an executive summary of European guidance for diagnosis of postmenopausal osteoporosis FRAX was recommended for fracture risk assessment [13]. This method, along with the Garvan algorithm [14, 15] and the Polish method, were used in the current study to establish fracture risk [16]. All three methods are available as on-line fracture-risk calculators.

The aim of the study was to verify the thesis that calcium intake influences the risk of osteoporotic fractures established by the above-mentioned on-line calculators. To our knowledge, such a study design has not been applied so far.

\section{Material and methods}

\section{Patients}

The presented analyses were performed in subjects selected from the database of females gathered in one outpatient osteoporotic clinic in the south of Poland, the "Gliwice Osteoporosis Study" (GO Study). The total number of registered patients is 2354 . After searching the database, 521 postmenopausal women aged over 55 years with available data on dietary calcium intake were enrolled. Mean age, height, weight, and BMI were $67.7 \pm 8.6$ years, $157.3 \pm$ $6.3 \mathrm{~cm}, 64.9 \pm 11.1 \mathrm{~kg}$, and $26.2 \pm 4.3 \mathrm{~kg} / \mathrm{m}^{2}$, respectively. At least one osteoporotic fracture was reported by 230 women, and 209 confirmed at least one major fracture (hip, spine, arm, or forearm). The following clinical risk factors were noted: hip fracture in parents in 25 , steroid use in 34, rheumatoid arthritis in 5, smoking in 56 , secondary causes of osteoporosis in 39 , and falls during last 12 months in 132 subjects. Among women with falls 82 had one fall, 20 had two falls, and 30 had three or more falls.

\section{Methods}

Fracture risk was established using FRAX (major and hip fractures, 10 years), the Garvan calculator (any and hip fractures, 5 and 10 years), and the Polish algorithm available at www.fracture-risk.pl (any fractures, 5 years). FRAX expresses the fracture probability within 10 years limited by life expectancy, and the two other algorithms express fracture risk. Briefly, the FRAX algorithm takes into account the following: age, weight, height, femoral neck T-score, and several clinical risk factors; the Garvan calculator includes age, the number of fractures after the age of 50 years, the number of falls in the last year, and femoral neck T-score; and the Polish algorithm takes into consideration steroid use, height, prior fracture after the age of 40 years, falls in last 12 months, and femoral neck T-score. $\mathrm{T}$-score values used for fracture risk calculations were derived from femoral neck BMD measurements, which were performed using a DPX device (Lunar, GE, USA). All scans were done by one experienced operator.

Calcium intake was established based on a dietary questionnaire [17].

\section{Statistics}

Statistical analysis was performed using Statistica software (StatSoft, Tulsa, OK, USA). Descriptive statistics for continuous variables were presented as mean values and standard deviations. The normality of data distribution was checked by the Shapiro-Wilk test. For comparative analysis Student's t-test for independent samples or the Mann-Whitney U-test (in the case of normal and abnormal distribution, respectively) were employed. Analysis of variance (ANOVA) and post hoc verification with the least significant difference (LSD) test were performed when comparisons among more than two subgroups were necessary. Correlation analysis was done using Spearman's correlation tests. Significance for results of all statistical analyses was assumed at $\mathrm{p}<0.05$

\section{Results}

The mean femoral neck T-score was $-2.03 \pm 0.78$. The following results of fracture risk assessment were obtained: FRAX for hip $3.25 \pm 3.83 \%$, FRAX for major fractures $8.76 \pm 5.8 \%, 5$ years Garvan for hip $9.25 \pm 14.72 \%$, 5 years Garvan for any fractures $18.06 \pm 15.7 \%, 10$ years Garvan for hip $15.5 \pm 20.7 \%$, 10 years Garvan for any fractures $31.8 \pm 22.3 \%$, and 5 years Polish algorithm for any fracture $16.13 \pm 9.9 \%$. Mean calcium intake was $755 \pm 310 \mathrm{mg}$ daily.

Average calcium intake did not differ between subjects with or without prior fracture. Also, in subjects with prior falls the calcium intake did not differ in comparison to that seen in those without falls (data not shown). However, when the analysis of calcium intake was performed in relation to the number of reported falls, some significant differences were found. Namely, frequent fallers (three or more reported falls) had significantly lower calcium intake in comparison to subjects with no falls or one fall. Detailed data are presented in Figure 1.

A tendency towards lower but not significantly different calcium intake was shown in subgroups with high fracture risk for hip by FRAX $(\geq 3 \%)$ versus low hip FRAX $(<3 \%)$. The same analysis for FRAX major fracture risk revealed a similar tendency with a slightly lower calcium intake in high $(\geq 20 \%)$ versus low $(<20 \%)$ fracture-risk groups, respectively. Mean values of calcium intake in cohorts stratified according to FRAX fracture probability categories are presented in Table 1. 


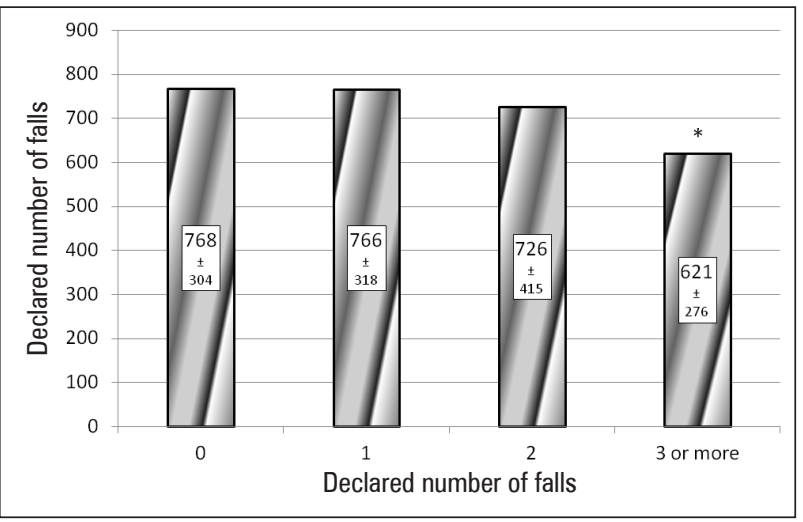

Figure 1. Average dietary calcium intake $($ mean $\pm S D)$ in subgroups stratified according to the declared number of falls. * Significantly lower calcium intake in comparison to subjects with no falls $(p<0.05)$ or subjects who declared 1 fall $(p<0.05)$; least significant difference (LSD) test

Similar analysis was performed for the Garvan algorithm. The group was stratified according the thresholds suggested by authors of the Garvan calculator. For five-year hip fracture risk the following cut-off points were applied: $<2 \%-$ low risk, suggesting no necessity for therapy; > 5\% - high risk, suggesting the necessity for therapy; and intermediate range $2-5 \%$ requiring an individual approach. Respective thresholds for five-year risk of any fractures were: $<3 \%, 3-9 \%$ and $>9 \%$; for 10 -year risk of hip fracture: $<8 \%, 8-13 \%$ and $>13 \%$; and for 10 -year risk of any fracture: $<14 \%, 14-26 \%$, and $>26 \%$. For the Polish method the same thresholds as for the Garvan five-year fracture risk for any fracture were applied. None of the above-mentioned comparisons showed any significant relation between fracture risk and dietary calcium intake. Detailed data about calcium intake for fracture risk categories according to the Garvan and Polish algorithms are presented in Table 1.

Concordantly, correlation analysis revealed no correlation between calcium intake and values for fracture risk obtained by any of the analysed calculators (data not shown).

\section{Discussion}

To the best of our knowledge, the current study is the first attempt to find possible relationships between dietary calcium intake and fracture risk estimation based on several calculators available on-line. We did not find that calcium intake influences the fracture risk, but some nonsignificant trends for risk established by FRAX calculator were observed. A tendency towards lower calcium intake was shown in subgroups with high hip-fracture risk by FRAX $(\geq 3 \%)$ versus low risk $(<3 \%)$ and in the subgroup with high major-fracture risk by FRAX $(\geq 20 \%)$ versus low risk $(<20 \%)$. In the case of the other two calculators, even such a weak tendency was not observed.

Only one previously published study presented the correlation between FRAX and calcium intake, and such a relationship was not observed [9]. However, the authors did not perform more detailed analysis for subgroups divided according to thresholds of fracture risk as we did. We consider that, despite the lack of clear evidence of the effect of calcium intake on fracture risk, our current results suggest that the role of calcium cannot be excluded. Probably the most important finding in the current study concerns the impact of calcium intake on fall rate. In women with three or more reported falls, calcium intake was significantly lower than in women without falls and with one fall only (see Fig. 1). Interestingly, only for FRAX we noted some nonsignificant differences in regard to calcium intake, whereas the results of the Garvan and Polish algorithms, both including falls as a fracture risk factor, did not reveal any dependence on calcium intake. It can therefore be hypothesized that the influence of calcium intake on functional status and its direct effect on skeletal status are at least partially independent of each other. It is also highly likely that a lot of unidentified confounders may modify the relationship between dietary calcium supply and fracture risk, explaining our and other authors' inconsistent observations. Further studies are necessary in order

Table 1. Average dietary calcium intake (mean $\pm S D)$ in subsequent fracture risk categories determined according to the analysed calculators (FRAX, Garvan, and the Polish algorithm)

\begin{tabular}{lccccccc}
\hline $\begin{array}{l}\text { Fracture risk/ } \\
\text { /probability } \\
\text { category* }\end{array}$ & FRAX major fx & FRAX hip fx & $\begin{array}{c}\text { Garvan 5-ys. } \\
\text { any fx }\end{array}$ & $\begin{array}{c}\text { Garvan 5-ys. } \\
\text { hip fx }\end{array}$ & $\begin{array}{c}\text { Garvan 10-ys. } \\
\text { any fx }\end{array}$ & $\begin{array}{c}\text { Garvan 10-ys. } \\
\text { hip fx }\end{array}$ & $\begin{array}{c}\text { Polish algorithm } \\
\text { 5-yrs. any fx }\end{array}$ \\
\cline { 2 - 8 } & $760 \pm 311$ & $765 \pm 299$ & $800 \pm 283$ & $730 \pm 287$ & $737 \pm 297$ & $748 \pm 290$ & N/A** \\
\hline Low & $\mathrm{N}^{*} \mathrm{~A}^{* * *}$ & $\mathrm{~N} / \mathrm{A}^{* * *}$ & $752 \pm 295$ & $781 \pm 304$ & $760 \pm 283$ & $859 \pm 357$ & $778 \pm 311$ \\
\hline High & $700 \pm 299$ & $744 \pm 328$ & $761 \pm 322$ & $759 \pm 332$ & $764 \pm 337$ & $740 \pm 323$ & $755 \pm 309$ \\
\hline
\end{tabular}

${ }^{*}$ the threshold values of subsequent categories for each of the analysed calculators are given in the text; ${ }^{*}$ only 3 subjects were classified in this category, so the data are not eligible for statistical analysis; ${ }^{* *}$ the "medium" fracture probability category was not applied for FRAX assessment tool 
to establish the potential role of calcium intake on fracture risk and falls.

A comparison between our current study and previously published ones provides an interesting observation regarding differences in calcium intake. In the former study, the calcium intake assessed in population-based females aged over 55 years was much lower [10] than in the current study. Probably the currently analysed group consisting of patients, and not recruited from healthy subjects, were prone to consuming more calcium, e.g. as a result of educational activities. We consider that the lack of differences for calcium intake in regard to fracture status and BMD noted in the current study might be biased by modification in dietary habits taken as secondary prevention, e.g. after a previous fracture.

The study has some limitations. The risk of fracture depends on long-term living habits, and calcium intake has been chosen as the only factor of its kind. Therefore, it can be stated that the dietary questionnaire cannot accurately assess lifetime calcium intake, which may affect the current results. Diet may influence bone status not only through calcium intake but also by its other components, such as protein, sodium, or phosphorus intake, and such potential additional factors were not studied. Moreover, the assessment carried out on the basis of a one-off questionnaire probably cannot replace the data collected during longitudinal observation. Vitamin D levels were not measured. Data on vitamin D supplementation, physical condition, comorbidities, and drugs, which could additionally modify skeletal status or incidence of falls, were not available for analysis. And finally, data presented in current study were gathered in patients in one outpatient clinic, and our study cohort cannot be considered as a population-representative sample. However, the large number of patients in the wide age range (55-90 years) and the high variability of the estimated calcium intake (250-1800 mg/day) allows a reliable summary of the results.

Concluding, calcium intake does not correlate with fracture risk established by calculators available on-line but low calcium intake may increase the risk of falls.

\section{References}

1. Zhu K, Prince RL. Calcium and bone. Clin Biochem. 2012; 45(12): 936-942, doi: 10.1016/j.clinbiochem.2012.05.006, indexed in Pubmed: 22609892.
2. Tai V, Leung W, Grey A, et al. Calcium intake and bone mineral density: systematic review and meta-analysis. BMJ. 2015; 351: h4183, doi: 10.1136/bmj.h4183, indexed in Pubmed: 26420598.

3. Khan B, Nowson CA, Daly RM, et al. Higher Dietary Calcium Intake Are Associated With Reduced Risks of Fractures, Cardiovascular Events, and Mortality: A Prospective Cohort Study of Older Men and Women. J Bone Miner Res. 2015; 30(10): 1758-1766, doi: 10.1002/jbmr.2515, indexed in Pubmed: 25828852

4. Vannucci L, Masi L, Gronchi G, et al. Calcium intake, bone mineral density, and fragility fractures: evidence from an Italian outpatient population. Arch Osteoporos. 2017; 12(1): 40, doi: 10.1007/s11657-017-0333-4, indexed in Pubmed: 28401496.

5. Warensjö E, Byberg L, Melhus H, et al. Dietary calcium intake and risk of fracture and osteoporosis: prospective longitudinal cohort study. BMJ. 2011; 342: d1473, doi: 10.1136/bmj.d1473, indexed in Pubmed: 21610048

6. Zhao JG, Zeng XT, Wang J, et al. Association Between Calcium or Vitamin D Supplementation and Fracture Incidence in Community-Dwelling Older Adults: A Systematic Review and Meta-analysis. JAMA 2017; 318(24): 2466-2482, doi: 10.1001/jama.2017.19344, indexed in Pubmed: 29279934

7. Harvey NC, Biver E, Kaufman JM, et al. The role of calcium supplementation in healthy musculoskeletal ageing: An expert consensus meeting of the European Society for Clinical and Economic Aspects of Osteoporosis, Osteoarthritis and Musculoskeletal Diseases (ESCEO) and the International Foundation for Osteoporosis (IOF). Osteoporos Int. 2016; 28(2): 447-462, doi: 10.1007/s00198-016-3773-6, indexed in Pubmed: 27761590.

8. Pham TT, Nguyen DN, Dutkiewicz E, et al. A profiling analysis of contributions of cigarette smoking, dietary calcium intakes, and physical activity to fragility fracture in the elderly. Sci Rep. 2018; 8(1): 10374, doi: 10.1038/s41598-018-28660-y, indexed in Pubmed: 29991706.

9. van den Berg P, van Haard PMM, van den Bergh JPW, et al. First quantification of calcium intake from calcium-dense dairy products in Dutch fracture patients (the Delft cohort study). Nutrients. 2014; 6(6): 2404-2418, doi: 10.3390/nu6062404, indexed in Pubmed: 24959951.

10. Włodarek D, Głąskka D, Kołota A, et al. Calcium intake and osteoporosis: the influence of calcium intake from dairy products on hip bone mineral density and fracture incidence - a population-based study in women over 55 years of age. Public Health Nutr. 2014 * 17(2): 383-389, doi: 10.1017/S1368980012005307, indexed in Pubmed: 23217270.

11. Jeong SH, Kim JS. Impaired Calcium Metabolism in Benign Paroxysmal Positional Vertigo: A Topical Review. J Neurol Phys Ther. 2019; 43(Suppl 2): S37-S41, doi: 10.1097/npt.0000000000000273, indexed in Pubmed: 30883492.

12. Huang TC, Cecchin FC, Mahoney $\mathrm{P}$, et al. Corrected OT interval (QTc) prolongation and syncope associated with pseudohypoparathyroidism and hypocalcemia. J Pediatr. 2000; 136(3): 404-407, doi: $10.1067 / \mathrm{mpd} .2000 .103447$, indexed in Pubmed: 10700702 .

13. Kanis JA, Cooper C, Rizzoli R, et al. Scientific Advisory Board of the European Society for Clinical and Economic Aspects of Osteoporosis and Osteoarthritis (ESCEO) and the Committees of Scientific Advisors and National Societies of the International Osteoporosis Foundation (IOF). Executive summary of European guidance for the diagnosis and management of osteoporosis in postmenopausal women. Aging Clin Exp Res. 2019; 31(1): 15-17, doi: 10.1007/s40520-018-1109-4, indexed in Pubmed: 30612282

14. Nguyen ND, Frost SA, Center JR, et al. Development of a nomogram for individualizing hip fracture risk in men and women. Osteoporos Int. 2007; 18(8): 1109-1117, doi: 10.1007/s00198-007-0362-8, indexed in Pubmed: 17370100.

15. Nguyen ND, Frost SA, Center JR, et al. Development of prognostic nomograms for individualizing 5 -year and 10 -year fracture risks. Osteoporos Int. 2008; 19(10): 1431-1444, doi: 10.1007/s00198-008-0588-0, indexed in Pubmed: 18324342

16. Adamczyk P, Werner A, Bach M, et al. Risk Factors for Fractures Identified in the Algorithm Developed in 5-Year Follow-Up of Postmenopausal Women From RAC-OST-POL Study. J Clin Densitom. 2018; 21(2) 213-219, doi: 10.1016/j.jocd.2017.07.005, indexed in Pubmed: 28826886.

17. Worksheet to Find Out How Much Calcium You Need Each Day. Department of Health, New York State. https://www.health.ny.gov/publications/1981/index.htm (28.12.2020.) 